OPEN ACCESS

Edited by:

Wei Guo,

Institute of Zoology (CAS), China

Reviewed by:

Lihua Huang,

South China Normal University, China

Aishwarya Swaminathan,

University of Massachusetts Medical

School, United States

Xubo Zhang,

Shanxi University, China

*Correspondence:

Junzheng Zhang

zhangjz@cau.edu.cn

Specialty section:

This article was submitted to Epigenomics and Epigenetics, a section of the journal Frontiers in Genetics

Received: 20 March 2020

Accepted: 15 June 2020

Published: 10 July 2020

Citation:

Mo $D$, Chen Y, Jiang N, Shen J and Zhang J (2020) Investigation of Isoform Specific Functions of the

$V$-ATPase a Subunit During

Drosophila Wing Development.

Front. Genet. 11:723

doi: 10.3389/fgene.2020.00723

\section{Investigation of Isoform Specific} Functions of the V-ATPase a Subunit During Drosophila Wing Development

\author{
Dongqing Mo, Yao Chen, Na Jiang, Jie Shen and Junzheng Zhang*
}

Department of Entomology and MOA Key Lab of Pest Monitoring and Green Management, College of Plant Protection, China Agricultural University, Beijing, China

The vacuolar ATPases (V-ATPases) are ATP-dependent proton pumps that play vital roles in eukaryotic cells. Insect V-ATPases are required in nearly all epithelial tissues to regulate a multiplicity of processes including receptor-mediated endocytosis, protein degradation, fluid secretion, and neurotransmission. Composed of fourteen different subunits, several V-ATPase subunits exist in distinct isoforms to perform cell type specific functions. The $100 \mathrm{kD}$ a subunit (Vha100) of V-ATPases are encoded by a family of five genes in Drosophila, but their assignments are not fully understood. Here we report an experimental survey of the Vha100 gene family during Drosophila wing development. A combination of CRISPR-Cas9 mutagenesis, somatic clonal analysis and in vivo RNAi assays is used to characterize the requirement of Vha100 isoforms, and mutants of Vha100-2, Vha100-3, Vha100-4, and Vha100-5 genes were generated. We show that Vha100-3 and Vha100-5 are dispensable for fly development, while Vha100-1 is not critically required in the wing. As for the other two isoforms, we find that Vha100-2 regulates wing cuticle maturation, while Vha100-4 is the single isoform involved in developmental patterning. More specifically, Vha100-4 is required for proper activation of the Wingless signaling pathway during fly wing development. Interestingly, we also find a specific genetic interaction between Vha100-1 and Vha1004 during wing development. Our results revealed the distinct roles of Vha100 isoforms during insect wing development, providing a rationale for understanding the diverse roles of V-ATPases.

Keywords: V-ATPase a subunit, wing development, Drosophila melanogaster, V-ATPase isoform, V100-2

\section{INTRODUCTION}

The vacuolar ATPases (V-ATPases) are ubiquitous proton pumps which play important roles in eukaryotic cells (Forgac, 2007). V-ATPases transport proton into intracellular compartments, and are therefore crucial for $\mathrm{pH}$ homeostasis in organelles such as endosomes, secretory vesicles, synaptic vesicles, and lysosomes (Hinton et al., 2009). The V-ATPases are generally required for a broad spectrum of cellular processes, including endosomal trafficking, lysosomal degradation, and exocytosis (Forgac, 2007). Insect V-ATPases are expressed in nearly all epithelial tissues and are well-known for their roles in physiological activities such as secretion of $\mathrm{K}+$ and 
$\mathrm{Na}+$ and formation of fluid (Wieczorek et al., 2009). Moreover, recent studies have illuminated the importance of V-ATPases for insect development. In Drosophila melanogaster, mutations that dampen the V-ATPases activity are reported to disrupt the formation of eye, wing and egg chambers (Yan et al., 2009; Buechling et al., 2010; Hermle et al., 2010; Vaccari et al., 2010). Further studies demonstrated that V-ATPases are involved in regulation of cell proliferation, cell fate determination and tissue patterning through modification of key developmental signaling pathways (Kobia et al., 2013; Gleixner et al., 2014; Portela et al., 2018; Ren et al., 2018).

The V-ATPase is a large protein complex composed of 14 different subunits that are organized into the cytosolic V1 region and the membrane-bound V0 region (Nelson, 2003). Region V1 hydrolyzes ATP and provides energy to pump protons through the protein lipid pores formed in region V0 (Jianhua et al., 2015). The $\mathrm{V} 1$ region contains eight subunits while the $\mathrm{V} 0$ domain is assembled by six different subunits (Forgac, 2007). The regulatory $\mathrm{C}$ subunit is located in the V1 domain and interacts with subunit a in the V0 domain (Wilkens et al., 2004; Inoue and Forgac, 2005; Cipriano et al., 2008). Therefore, the $\mathrm{C}$ subunit is well positioned to control the reversible dissociation of the $\mathrm{V}$-ATPase complex (Inoue et al., 2005; Forgac, 2007). The C subunit is encoded by Vha44 in Drosophila, which is required for endolysosomal acidification and regulates elimination of nurse cells in the ovary (Mondragon et al., 2019), cell competition in the eye imaginal disk (Nagata et al., 2019), and apical endocytosis in the wing disk epithelial cells (Gleixner et al., 2014). Ectopic Vha44 expression is shown to impair endolysosomal degradation and induce invasive cell behavior in the developing wing disk (Petzoldt et al., 2013) as well as differentiation defects in the eye disk (Portela et al., 2018).

Many of the V-ATPase subunits exist in multiple isoforms which are often expressed in a cell type specific manner (Toei et al., 2010). In Drosophila, the V-ATPase multigene family consists of 33 different genes (Julian, 1999; Allan et al., 2005). Apart from five subunits in the $\mathrm{V} 1$ region and the accessory subunits VhaAC45 and VhaM8.9, other V-ATPase subunits are encoded by two to five genes (Allan et al., 2005). In vertebrates, isoforms of subunit a in the V0 domain contain information necessary for targeting the V-ATPase complexes to the appropriate plasma membrane (Toyomura et al., 2003; Qi et al., 2007; Saw et al., 2011). In Drosophila, the V-ATPase a subunit is encoded by Vha100-1, Vha100-2, Vha100-3, Vha1004, and Vha100-5 with specific tissue distribution patterns (Toei et al., 2010). Previous studies have suggested that Vha100-1 is an isoform required for synaptic vesicle exocytosis in the nervous system (Hiesinger et al., 2005). Loss of Vha100-1 leads to vesicle accumulation in synaptic terminals (Wang et al., 2014), neuronal degeneration (Williamson et al., 2010a), and defects in brain wiring (Williamson et al., 2010b). RNAi knock-down experiments indicate that Vha100-2 is involved in regulation of neural stem cells proliferation (Wissel et al., 2018), acid generation of the midgut (Overend et al., 2016), elimination of nurse cells in the ovary (Mondragon et al., 2019), and cell competition in the eye disk (Nagata et al., 2019). Similar as Vha100-2, knock-down of Vha100-4 also leads to acidification defect in the larval midgut (Overend et al., 2016). The roles of
Vha100-3 and Vha100-5 are still unclear, and our understanding of whether and how Vha100 isoforms collaboratively regulate the development of specific tissue is incomplete.

In order to further investigate the functional diversity of the $\mathrm{V}$-ATPase a subunit isoforms, we generated and characterized mutants of Vha100-2, -3, -4, and -5. We found that among the five isoforms, Vha100-3 and Vha100-5 are dispensable for fly development. We further demonstrated that Vha100-2 is specifically required for wing cuticle formation, while Vha100-4 is involved in Wingless signaling activation. Comparative studies revealed that Vha100-1 and Vha100-4 execute both independent and redundant function during fly wing development. Our studies uncovered the isoform specific functions of the V-ATPase a subunit during Drosophila wing development.

\section{MATERIALS AND METHODS}

\section{Fly Genetics}

Fly stocks and all fly crosses were maintained at $25^{\circ} \mathrm{C}$ on standard fly food. The following fly stocks were used: hh-Gal4, UAS-mCD8-gfp/TM6B (Ren et al., 2018); Vha1002 RNAi (TH04790.N; TsingHua Fly Center); FRT42D, Vha44 ${ }^{K G 00915} /$ Cyo (\#111534; Kyoto Stock Center); FRT42D, Vha44 ${ }^{\mathrm{K} 05440} /$ Cyo (\#111081; Kyoto Stock Center); FRT82B, Vha100-1 $1^{1}$ TM3, Sb (\#39669; Bloomington Drosophila Stock Center); w;Sco/Cyo (\#2555; Bloomington Drosophila Stock Center); and w;TM3/TM6B (\#2537; Bloomington Drosophila Stock Center). The Ubx-FLP; FRT82B, Ubi-RFP/TM6B, Ubx-FLP; FRT42D, Ubi-RFP/Cyo, and Ubx-FLP; FRT42D, Ubi-GFP/Cyo stocks were used to generate mosaic mutant clones in the wing disks (Ren et al., 2018). The sens-GFP reporter was described before (Sarov et al., 2016) and obtained from Bloomington Drosophila Stock Center (\#38666). The $f z 3$-LacZ reporter was described before (Sato et al., 1999).

\section{CRISPR-Cas9 Mediated Mutagenesis}

The sgRNA targets were designed against the genomic sequences of Vha100-2, Vha100-3, Vha100-4, and Vha100-5 with CRISPR Optimal Target Finder ${ }^{1}$ (Gratz et al., 2014). Templates for sgRNA transcription were generated by annealing of two DNA oligonucleotides and subsequent PCR amplification (Bassett et al., 2013). In vitro transcription was performed with the T7 RiboMAX ${ }^{\mathrm{TM}}$ Kit (Promega, P1320) and the sgRNAs were purified by phenol-choloroform extraction and isopropanol precipitation. Cas 9 mRNA was transcribed with the mMESSAGE mMACHINE ${ }^{\circledR}$ T7 Transcription Kit (Ambion), using a linearized plasmid containing the Cas9 cDNA (Addgene plasmid 42251) as template. The Cas9 mRNA were polyadenylated with the Escherichia coli Poly(A) polymerase Kit (NEB), and purified with the RNeasy Mini Kit (QIAGEN). $15 \mu \mathrm{g}$ of Cas9 mRNA and $7.5 \mu \mathrm{g}$ sgRNA were mixed with DEPC water in a $30 \mu \mathrm{l}$ volume for embryo injection. Fly embryos of the $w^{1118}$ strain (\#5905; Bloomington Drosophila Stock Center) were injected using standard protocols by Fungene Biotech (Beijing, China).

\footnotetext{
${ }^{1}$ http://tools.flycrispr.molbio.wisc.edu/targetFinder/
} 
Vha100-1\% 1 . . . . . . . - MGSLFRSEEMALCQLFLQSEAAYACVSELGELGLVQFRDLNPDVNAFQRKFVNEVRRCDEMERKLRYL 68 Vha100-2 1 . . . . . . . . MGDMFRSEEMALCQMFI QPEAAYT SVSELGETGCVQFRDLNVNVNAFQRKFVTEVRRCDELERKIRYI 68 Vha100-3/ 1 MR - - VFKKRQTKVKSFFRSEDMDLCQLLLHTENAFDCL I EVGHHGAVQFNNVYDEDRLLNNLYSKKVTQCYELLRIVDSL 78 Vha100-4/. 1 MSKWWSCGSNQESNS I FRSEVMSLVQMYLQPEAAYDT I AALGEVGCVQFRDLNAK I NAQQRKF I GEVRRCDELERR IRYV 80 Vha100-5/ 1 . . . . . . . . . - MGDMFRSEKMALCQLF I QPEAAYAS I AELGEKGCVQFRDLNEEVSAF QRKYVNEVRRCDDMERRLRYV 68

Vha100-1\% 69 EKEIK - - -KDGI PMLD - - TGESPEAPQPREMIDLEATFEKLENELREVNQNAEALKRNFLELTEL - . . . . - - KH I LRKTQ 136 Vha100-2 69 ETEIK - - -KDG I VLPDI Q-DD I PRAPNPREI I DLEAHLEKTESEMI ELAQNEVNMKSNYLELTEL - . . . . - - RKVLENTQ 137 Vha100-3/ 79 HTY I VQLHVNE I FYPDVDRE . . . . . . . . . . - NRLKEKDL AKY SDSLKR I HVEASAVTEHYYRLDSRRNRMMEHSF ALNKAN 148 Vha100-4/ 81 TAELN - . - KEGHKVLDLM -DDFPPAPQPREI I DLELHLEKTETEI LELAANNVNLQT SYLEL SEM - . . . . . . I QVLERTD 149 Vha100-5/ 69 ESEMK - - -KDEVKL PVLRPEEEP I APNPRE I VDLEAQLEKTDNELREMS ANGASLDANFRHMQEL - . . . . . - KYVLENTE 138

Vha100-1/ 137 VFFDESVPTVYKSSGAYSSSKYRRYPQMADNQNEDEQAQLLGEEGVRASQPGQNLKLGFVAGVILRERLPAFERMLWRAC 216 Vha100-2/ 138 GFF SDQEVLNLDSS - . . . . . . . . . . . . . . . . . . . - NRAGGD - . - NDAAAQHRGRLGFVAGV INRERVFAFERMLWRIS 191 Vha100-3/ 149 KYMVSDM - . - - GSELLYS - . . . . . . . . . . . . . - ESTVI GL VQDATTT SGAYPAHLNYMI GCIRADKFYSFELLLYRLC 207 Vha100-4/ 150 QFF SDQESHNFDLN - . . . . . . . . . . . . . . . . . . . . KM - - GT - . - HRDPEKSNGHLGF VAGVI SREREYAFERMLWRIS 201 Vha100-5/ 139 GFF SDQEVI INLDVN . . . . . . . . . . . . . . . . . . . . . RKLDPEDPANLPGAAQRGQLAFVAGVIKLERFF SFERMLWRIS 195

Vha100-1/ 217 RGNVFLRQAMI ETPLEDPTNGDQ - . - VHKSVFI IFFQGDQLKTRVKK I CEGFRATLYPCPEAPADRREMAMGVMTRIEDL 293 Vha100-2/192 RGNVFLKRSDLDEPLNDPATGHP . . - I YKT VFVAFFQGEQLKNR I KKVCTGFHASLYPCPSSHNEREEMVRNVRTRLEDL 268 Vha100-3/' 208 SFNL I I RF SEMP SPVYEYHYGYKPERVRKF A I LMMASSTM I WPKVLK I CAHYHVN I YDCPSSA SQREDKVKEL SQE I VNV 287 Vha100-4/' 202 RGNVFVRRCDVDVALTDPKTGNV - - - LHKSVFVVVFFQGDQLQARIRKVCTGFHAHMYPCPSSHSERQEMVKNVRTRLEDL 278 Vha100-5/' 196 RGN I FLRRAD I DGL VADEETGRP - . - VLKTVFV VAFFQGEQLKQR I KKVCTGYHAAVYPCPSSHAERKEM I KDVNVRLEDL 272

Vha100-1/ 294 NTVLGQTQDHRHRVLVAAAKNLKNWF VKVRK I KA I YHTLNLFNL - - - DVTQKCL I AECWVPLLDI ET I QLALRRGTERSG 370 Vha100-2 269 KL VL SQTEDHRSRVLATVSKNL P SWS I MVKKMKA I YHTLNL FNM - - - DVTKKCL I GECWVPTNDL PVVQKALSDGSAAVG 345 Vha100-3/' 288 EKVLKEAELMRRQI LEVAGRDLF I I RVNLRKALKVYDLMNRLRLVGGVEVPRYLLAEVY I PSSDVPEVEVI LRNASR I SG 367 Vha100-4/ 279 QVI I NQT SDHRTCVL QAALKQLPTWS AMVKKMKG I YHTLNLFNV - . - DLGSKCL I GEGWVPKRELLLVEVALAAGSASVG 355 Vha100-5/' 273 KLVLSQS ADHRSRVLNSA SKHLPRWS I MVRKMKA I YH I LNFFNP - - - DVTGKCL I GEGWVPTND I STVQDALARASK I SE 349

Vha100-1/ 371 SSVP - . . . . PIL - . . . . . . . . . . . . . . . . . . . . . . . . .RMQTFENPPTYNRTNKFTKAFQAL I DAYGVASYR 412 Vha100-2 346 ST IP - . . . . SFL . . . . . . . . . . . . . . . . . . . . . . . . . . . . . . NV I DTNEQPPTFNRTNKF TRGF QNL I DAYGVASYR 387 Vha100-3/' 368 GADN I DSSDEDEMNDMKTMPNT TPYP I EADF QPLEDMS AGA I LLKKNRL VNHMPPTYYFRLNKF TRGF QNL I DAYGMADYK 447

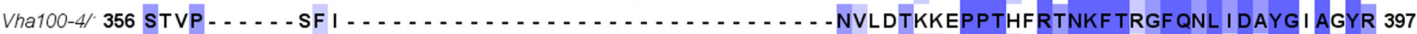

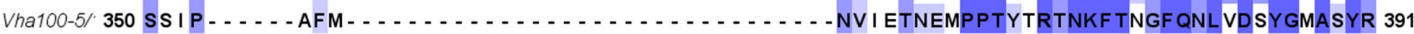
II

Vha100-1/ 413 EMNPAPYT I I TFPFLF AVMF GDL GHGA I MALFGLWMI RKEKGLAA _ . . - QKTDNE I WN IFFGGRY I IFLMGVFSMYTGL I 488 Vha100-2 388 ECNPALYTC I TFPFLFAVMF GDL GHGL I LVLF FAWMVLCERKLAR - . - - I RNGGE I IN I FFGGRY I I LL MGLFAMYTGLV 463 Vha100-3/ 448 ELNPAPYT I I TFPF LF AVMF GDL GHG I LL I LF S SLM IWKHRE I EKYQ I NAT SENE I LN I LYAGRY I I LLMGVFSVYMGLV 527 Vha100-4/ 398 EVNPGLYTC I TFPF LFAVMF GDMGHGT I LFLLGLWMV I DEKRLSK - . . . - KRGGE I WN I FFAGRYI I ML MGLFAMYTGFH 472 Vha100-5/ 392 EVNPALY YAC I TFPFLF AVMF GDL GHGL I LLLFASWL I I KEKQLSS - . - - I K - - EE I FN I FFGGRY I IFLMG I FS I YTGF I 465

Vha100-1/ 489 YNDI F SKSLNIFGSHWHL SYNKSTVMEN - -KF - LQLSPKGDYEGAPYPFGMDP I WQVAGANK I I FHNAYKMK I S I I FGV I 565 Vha100-2 464 YNDVF SKSMNLFGSRWF NNYNTTTVLTN - - PN - LQLPPNSS - AVGVYPF GMDPVWQLA - DNK I I FLNSFKMKL SI I FGVL 538 Vha100-3/' 528 YN I VMAKGF NLFGSSWSCRYNETTVYDPAFHVTLDSSHPHFY SGHPYPL GMDPVWAVCGQDSI TTTNSLKMKMAIVLGIS 607 Vha100-4/ 473 YND I F SKS I NVF GTRWVNVYNRTTVLTN - - PT - LQLNPSVA - TRGVYPMG I DP IWQSA - SNK I I FLNTYKMKL S I I FGVL 547 Vha100-5/ 466 YNDVF SKSMN I FGS AWHMNYTRDVVEDENLKY - I TLRPNDT - VYKTYYFF GMDP I WQL A - DNK I I FLNTFKMKL S I VGV I 542 IV

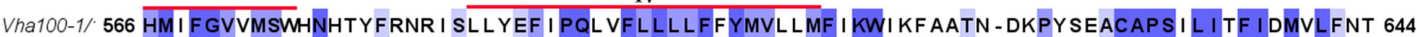
Vha100-2/ 539 HMVF GVCMSVVNF THFKRYAS I FLEFVPQI LFLLLLF GYMVFMMFFKWF SYNART SF QPETPGCAPSVL I MF I NMMLFKN 618 Vha100-3/' 608 QMMF GL GL AAANCVLLMNRKADL I LVV I PQM I FMLCL F GYL VFL I FYKMMSYGGHK - PAPYNAACAP SVL I TF I NMML MKK 686 Vha100-4/. 548 HMVF GVCMSVENFVFFKKYAY I I LQFVPQVLFLLLMF GYMCFMMFYKWVKYSPTTDVEADTPGCAPSVL I MF I DMVLFKT 627 Vha100-5/ 543 HM I FGVSMSVVNFAYYKKYAS I FLEFLPQVLFLLLLFGYMVFMMFFKWVVYNDTV - EGPL SPACAPS I L I LF I INM I LQGS 621

Vha100-1/ 645 PKPPPENCETYMFMGQHF I QVLFVLVAVGCI PVMLLAKPLLI MQARKQANVQP I AGAT - . . . . . . . . - SDAE - AGGVSN 712 Vha100-2 619 TEP - PKGCNEF MFE SQPQL QKAF VL I ALCC I PWMLL GKPL Y I KF TRKNKAHANH - - NGQL TGN I EL AEGETPL - PTGF SG 694 Vha100-3/ 687 EDP - VENCLDYMYPNERMI EF ALVG I AFCT I P I LLAGKP I YLMRRRRKMQQERERDFKRMRRQT I AEMRSTMRYTDDDNS 765 Vha100-4/ 628 ETA - LPGCDVNMFP I QKNLEMI FLVVALLCI PWI LLGKPLYIKYQRRNRPAGPVEEVDEI VEK I EVTTGKEI I - I TEV - A 704 Vha100-5/' 622 QDT - PEPCKEFMFDGQKS I QQVFVVVAI I CI PUMLLGKPLYIMIKRKTNGAPPP . . . . . . . . . . . . . . . . . . . . 674

VI

Vha100-1/ 713 SGSHGGGGGHEEEEEL SE I FI HQS I HT I EYVLGSVSHTASYLRLWAL SL AHAQLAEVLWT MVLS I GLKQE - - GPVGGIVL 790 Vha100-2 695 NEENAGGAHGHDDEPMSE I Y I HQA I HT I EYVL ST I SHTASYLRLWAL SL AHAQL SEVLWQMVL SL GLKMS - - GVGGA I GL 772 Vha100-3/ 766 ET SRQKSVDNEEEHEMSE IWI HSGI HT I ETVLGSVSHTASYLRLWAL SL AHDQL SDVLWHMVLTKGF ANTLPLYYGVPVL 845 Vha100-4/ 705 EAHESGGHSEEDDEPMSE IWI HQA I HT I EY I LST I SHT ASYLRLWAL SL AHAQL SEVLWTMVL AMGL QMN - - GYVGA I GL 782 Vha100-5/. 675 KPQSGGGEGHGEDDEMGE I FI HQAI HT I EYVLST VSHTASYLRLWAL SL AHAQL SEVLWNMVF SMGFKYD - - SY I GGILI 752

Vha100-1/ 791 TCVFAFWA I LTVGI LVLMEGL SAFLHTLRLHWVFQSKFYKGQGYAFQPFSFDAI I ENGAAAAEE Vha100-2/773 FI I FGAWCLFTLA I LVLMEGL SAFLLHTLRLHWWEFMSKFYEGMGYAFQPF SFKAILDGEEEE - . Vha100-3/ 846 MATFF AWA I LTVA I LVMMEGL SAF LHTLRLHWWEFQSKFFGGAGESFKAFNFPT SNQRS - . - . . Vha100-4/ 783 FF I FAVWEFFT I A I MVMMEGL SAFLHTLRLHWVEFMSKFYVGNGYPFTPFSFKD I L I VVEDD - . . Vha100-5/ 753 YVFFGAWALLTVGILVL IEGL SAFLHTLRLHWVEFMSKFYEGAGYAFEPFAFKT ILDVSEDD - . - 
FIGURE 1 | Amino acid sequences and structures of V-ATPase subunit a isoforms in Drosophila. Alignment of the five Drosophila V-ATPase a subunit isoform sequences (Vha100-1, Vha100-2, Vha100-3, Vha100-4, and Vha100-5) is shown. Identical residues are indicated by blue shades and similar residues are labeled by light purple shades. The seven putative transmembrane helices predicted from topographical analysis are shown with a red bar. The residues whose mutation has a significant effect on activity or assembly of the V-ATPase are indicated by asterisks. GenBank accession numbers assigned to Vha100-1, Vha100-2, Vha100-3, Vha100-4, and Vha100-5 are AAF56861, AAF55551, AAM68427, AAF55550, and AAF53116, respectively.

TABLE 1 | The sequence identity (and similarity) between pairs of Drosophila a subunit isoforms.

\begin{tabular}{|c|c|c|c|}
\hline Pairs of isoforms & Identity and (similarity) & Pairs of isoforms & Identity and (similarity) \\
\hline Vha100-1 and Vha100-2 & $58 \%(71 \%)$ & Vha100-2 and Vha100-4 & $65 \%(79 \%)$ \\
\hline Vha100-1 and Vha100-3 & $36 \%(51 \%)$ & Vha100-2 and Vha100-5 & $65 \%(79 \%)$ \\
\hline Vha100-1 and Vha100-4 & $53 \%(67 \%)$ & Vha100-3 and Vha100-4 & $33 \%(51 \%)$ \\
\hline Vha100-1 and Vha100-5 & $57 \%(71 \%)$ & Vha100-3 and Vha100-5 & $35 \%(53 \%)$ \\
\hline Vha100-2 and Vha100-3 & $35 \%(54 \%)$ & Vha100-4 and Vha100-5 & $56 \%(72 \%)$ \\
\hline
\end{tabular}

Males developed from the injected embryos (G0) were outcrossed to virgin females of TM3/TM6B (for Vha100-2 and Vha100-4) or Sco/Cyo (for Vha100-3 and Vha100-5). Single G1 males were each crossed to $4-5$ females of the corresponding balancer stocks and the progenies (G2) bearing the same balancer chromosome were maintained as an independent stock. About 50 G2 stocks were established from each injection, and mutations were screened by PCR test of genomic DNAs. Primer pairs were designed to generate PCR products covering the target sites, which were compared with the control sequences amplified from $w^{1118}$ genomic DNA. The primers used are: V100-2F: AACGTTGTCGTTGGCTGAAGCA; V100-2R: ATGT CATCCTGGATGTCGGGCA; V100-3F: CTGCGCATCGTGG ACAGTCTG; V100-3R: GAATGATCAGGTTGAAGGAGC; V100-4F: GCTGTGCTCCGAAAGTGAG; V100-4R: ACCT TGTGACCCTCCTTGTT; V100-5F: CAGTTATAACACTCG ATTTGA; and V100-5R: TTGAGTTCTTGCATGTGCCGGA. Mutant alleles were identified and named as Vha100-2 $2^{D 2}$, Vha100-3 $3^{D 3}$, Vha100-4 $4^{D 4}$, and Vha100-5 ${ }^{D 5}$. FRT82B (\#2035; Bloomington Drosophila Stock Center) was recombined into the Vha100-2 $2^{D 2}$ and Vha100-4 ${ }^{D 4}$ mutant genomes by standard genetic crosses for further mosaic analysis.

\section{mRNA in situ Hybridization in Drosophila Wing Imaginal Disks}

The coding regions of Vha100-2 (1208 bp-1430 bp of GeneBank \#AAF55551) and Vha100-4 (982 bp-1240 bp of GeneBank \#AAF55550) were used to generate antisense RNA probes for in situ hybridization. An autofluorescent alkaline phosphatase substrate (Vector) was used to visualize mRNA in the rhodamine channel, and mutant clones were marked by immunofluorescence staining of GFP protein as described before (Su et al., 2011).

\section{Immunofluorescence Staining}

Wing disks dissected from third-instar larvae were fixed in $4 \%$ paraformaldehyde for $15 \mathrm{~min}$, blocked in PBS containing 0.1\% Triton $\mathrm{X}-100$ and $0.2 \%$ BSA for $1 \mathrm{~h}$, and incubated overnight at $4^{\circ} \mathrm{C}$ with the following primary antibodies: mouse anti-Cut (1:100; 2B10; and DSHB), mouse anti-Wingless (1:200; 4D4; and
TABLE 2 | Summary of CRISPR-Cas9 sgRNA sequences.

\begin{tabular}{lll}
\hline $\begin{array}{l}\text { Gene } \\
\text { symbol }\end{array}$ & sgRNA-1 sequence & sgRNA-2 sequence \\
\hline Vha100-2 & GATGTTCCGTAGTGAGGAGA & GTATACCTCCGTATCTGAGC \\
Vha100-3 & GATTCGACGACTGTCCAGT & GCACAGCTTCGCTTGAACA \\
Vha100-4 & GCAAATGTATCTGCAGCCGG & GTTGCCGCCTTGGGGAGGT \\
Vha100-5 & GGGCGTAGGCTGCCTCCGGC & GTTCGCGATCTGAACGAGG \\
\hline
\end{tabular}

DSHB), mouse anti-Notch intracellular domain NICD (1:200; C17.9C6; and DSHB), mouse anti- Notch extracellular domain NECD (1:200; C458.2H; and DSHB), mouse anti-Dl (1:200; C594.9B; and DSHB), rabbit anti-GFP (1:2000; A11122; and Thermo Fisher), and rabbit anti-Caspase3 (1:200; Cell signaling). Alexa fluor-conjugated secondary antibodies (1:400; Invitrogen) were used. Alexa fluor-568 conjugated phalloidin was used to label cell morphology (1:200; Thermo Fisher). The fluorescence images were acquired with Leica SP8 confocal microscope and processed in Photoshop and ImageJ.

\section{Eosin $Y$ and FB28 Staining and Microscopy of the Adult Wing}

Adult flies with correct genotypes were collected and fixed overnight in isopropanol. Dissected adult wings were mounted in Euparal mounting media (BioQuip). For Eosin Y and FB28 staining experiments, 2 days old files were fixed in formaldehyde phosphate buffer, washed several times with PBS, and stained with FB28 solution $(1 \mathrm{mg} / \mathrm{ml}$; Sigma-Aldrich) at room temperature for $1 \mathrm{~h}$ or $0.5 \%$ Eosin $\mathrm{Y}$ at $55^{\circ} \mathrm{C}$ for $35 \mathrm{~min}$. Adult wings were then dissected and mounted in $80 \%$ glycerol. The images were captured with a Leica DMIL inverted microscope equipped with a QICAM Fast 1394 digital camera.

\section{RESULTS}

\section{Vha100 Isoforms Are Differentially Required for Fly Development}

The a subunit of the V-ATPase is encoded by five genes in Drosophila, which are spread at different locations throughout 

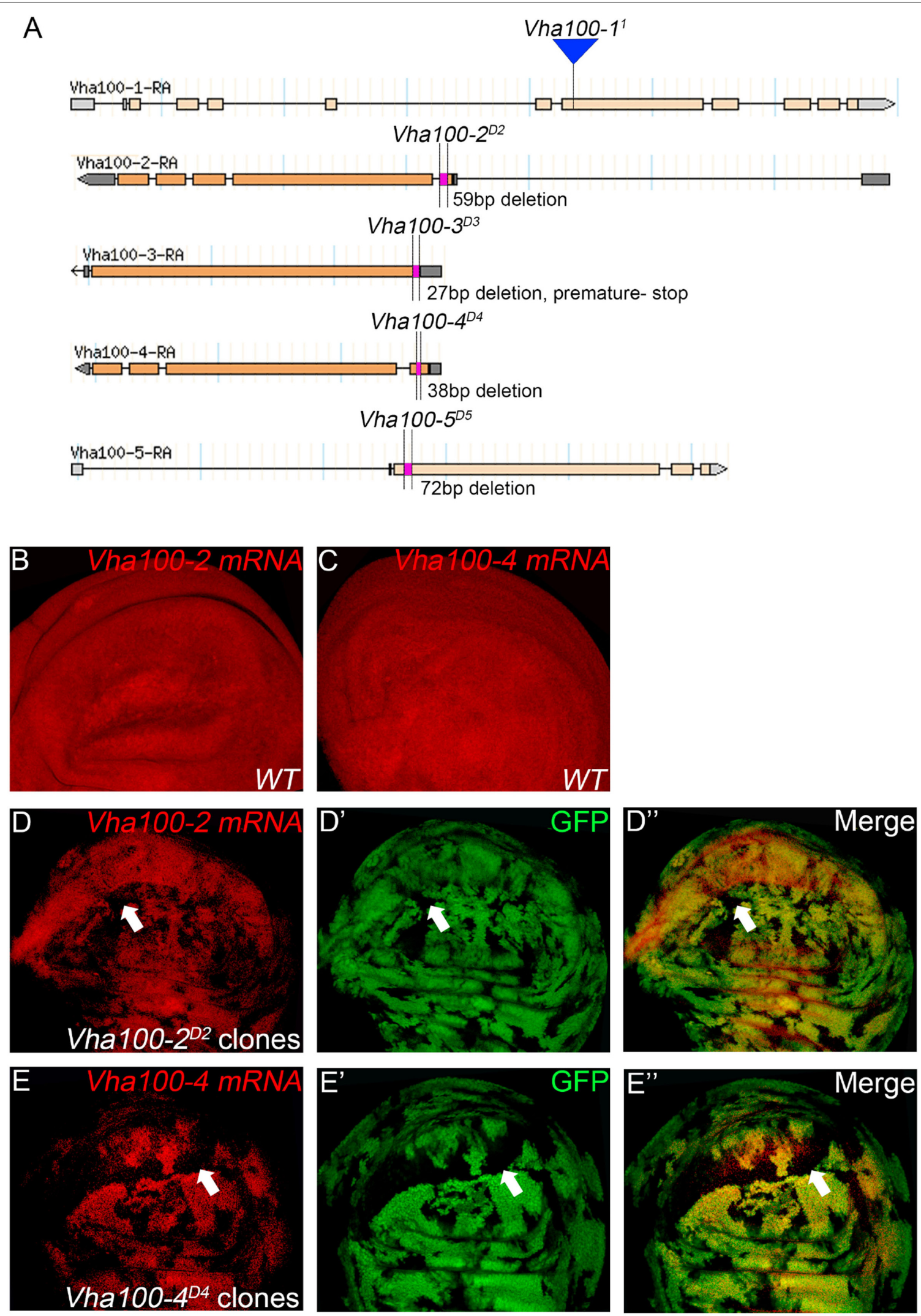
FIGURE 2 | Generation and verification of Vha100 mutants. (A) Schematic illustration of Vha100 mutant alleles. The Vha100-11 is a loss of function allele caused by mutation in the splice acceptor site. CRISPR-Cas9 mediated deleterious alleles are generated for other members of the Vha100 family. The position and molecular nature of each allele is labeled. (B-E) In situ hybridization in Drosophila wing imaginal disks. In wild type wing disks, Vha100-2 (B) and Vha100-4 (C) are ubiquitously expressed. In Vha100-2 $2^{2}$ mutant clones, the mRNA levels (red) are significantly down-regulated (D). The mRNA levels of Vha100-4 (red) are significantly down-regulated in Vha100-4 ${ }^{D 4}$ clones $(\mathbf{E})$. The down-regulation of Vha100-2 $(n=11)$ and Vha100-4 $(n=14)$ in mutant clones is fully penetrant. The mutant clones are marked by absence of GFP. Representative mutant clones are indicated by arrows. Scale bars: $10 \mu \mathrm{m}$.
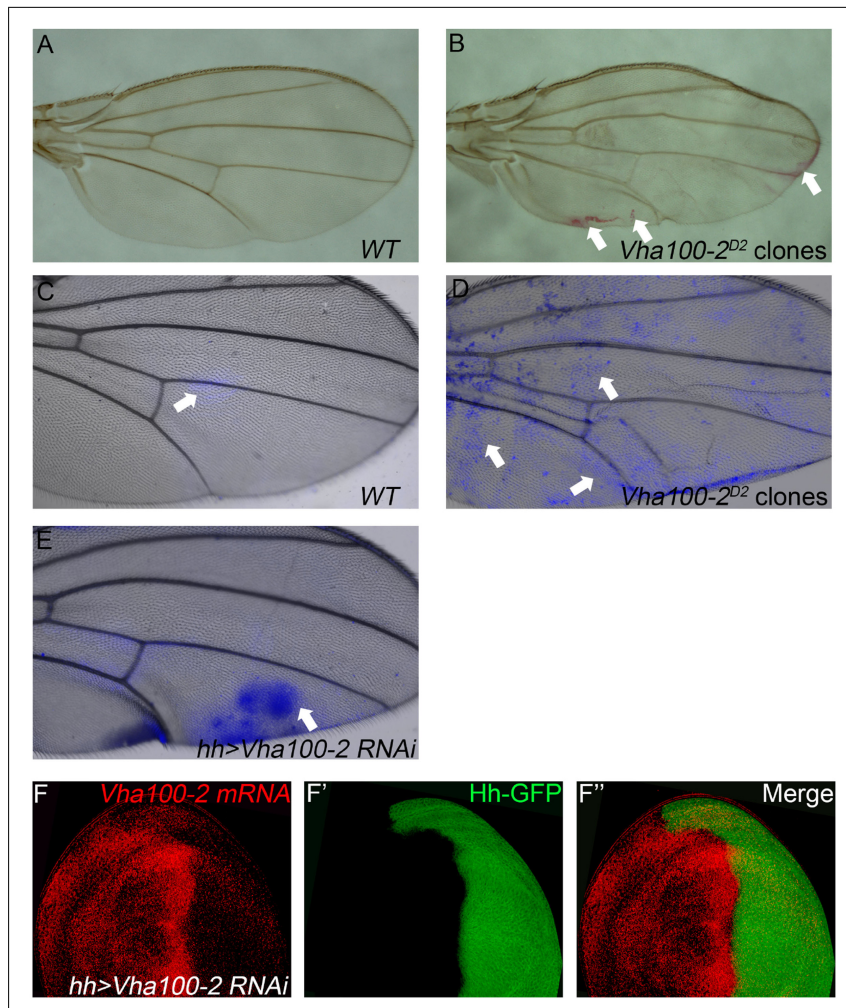

FIGURE 3 | Vha100-2 is specifically required for wing cuticle formation. (A,B) Penetration of Eosin $Y$ through the wing cuticle. The adult wing of Ubx-FLP; FRT82B, Ubi-RFP stock is used as wild type control, which is resistant to Eosin $Y$ penetration (A). By contrast, patches with Eosin $Y$ staining (red) are observed in fly wings harboring homozygous Vha100-2 D2 mutant clones (B) (C-E) Penetration of FB28 through the wing cuticle. In wild-type control wings, the FB28 dye (blue) shows limited penetration competency (C). The FB28 dye (blue) scatters into the wing bearing homozygous Vha100-2D2 clones (D). In Vha100-2 RNAi expression area, the penetration of the FB28 dye (blue) is evident (E). (F) The mRNA level of Vha100-2 (red) are down-regulated in RNAi expressing cells (marked by GFP). Representative Eosin $Y$ and FB28 staining patches are indicated by arrows. The down-regulation of Vha100-2 in RNAi expressing region is fully penetrant $(n=7)$. Scale bars: $0.5 \mathrm{~mm}$ in $\mathbf{( A - E )}$ and $10 \mu \mathrm{m}$ in $\mathbf{( F )}$.

the genome (Supplementary Figures S1A-D). The -1, -2, -3, -4 , and -5 isoform comprises $855,834,904,844$, and 814 amino acid residues, respectively. A hidden Markov model (Krogh et al., 2001; http://www.cbs.dtu.dk/services/TMHMM-2.0/) predicated that these isoforms have closely similar structures with seven putative transmembrane regions, which are conserved among the five isoforms (Figure 1). High conservation is also evident for the hydrophilic amino and hydrophobic carboxy terminals of fly Vha100 isoforms (Figure 1). Detailed analysis of sequence homology reveals that Vha100-3 is the most diverse member of the family, while the other isoforms share a similarity about 70\% between each other (Table 1). A number of residues of the a subunit have been experimentally demonstrated to be important for the activity or assembly of V-ATPase complex in yeast (Leng et al., 1996, 1998), which were later found to be conserved in mouse orthologs (Nishi and Forgac, 2000; Toyomura et al., 2000). All of these residues are conserved in the fly isoforms, with the exception of L800 (the Vha100-1 numbering), which is conserved in -3 and -5 but is a phenylalanine in -2 and -4 (Figure 1). It is likely that the less conserved regions may render different functions of Vha100 isoforms. Construction of a phylogenetic tree (Minh et al., 2013; Trifinopoulos et al., 2016) using the mouse, fly and yeast sequences reveals that the development of multiple isoforms of the a subunit appears to have occurred independently in these species (Supplementary Figure S1E).

Previous studies have isolated several Vha100-1 mutant alleles and revealed a neuronal specific role of Vha100-1 during fly development (Hiesinger et al., 2005). For the other four isoforms, mutagenesis analyses have not been reported yet. To better understand how Vha100 isoforms function in different developmental contexts, we generated Vha100-2, Vha100-3, Vha100-4, and Vha100-5 mutants by CRISPR-Cas9 mediated genome editing (Table 2). Mutations with small deletions were identified by genomic DNA PCR (Supplementary Figure S2) and used for further analysis (Figure 2A). Homozygous Vha100-3 $3^{D 3}$ and Vha100-5 $5^{D 5}$ mutant flies are viable and fertile with normal appearance of body structure and tissue morphology. Previous studies have shown that the expression of Vha100-3 is restricted to the testes in adult males, while the mRNA of Vha100-5 is undetectable by in situ hybridization in fly larvae (Wang et al., 2004; Allan et al., 2005). We reason that these two isoforms might perform specific physiological roles, but are dispensable for fly development. Therefore, we moved on to examine the roles of Vha100-1, Vha100-2, and Vha100-4 in fly wing development.

As Vha100-1 ${ }^{1}$ (Hiesinger et al., 2005), Vha100-2 ${ }^{D 2}$, and Vha100-4 $4^{D 4}$ are homozygous lethal mutants, somatic mosaic clones were generated using the FLP-FRT system to examine their roles in wing development. The FLP recombinase catalyzes exchange of the homologous chromosome arms and induces the generation of homozygous mutant cell clones with randomized size and location (Xu and Rubin, 1993). Importantly, the wild type cells surrounding the mutant clones serve as rigorous internal control for developmental studies (Theodosiou and $\mathrm{Xu}$, 1998). Taking advantage of the FLP-FRT system, we evaluated whether Vha100-2 $2^{D 2}$ and Vha100-4 $4^{D 4}$ (Figure 2A) behave as loss-of-function alleles by examining their mRNA levels in mutant mosaic clones. Both Vha100-2 (Figure 2B) and Vha100-4 (Figure 2C) were ubiquitously expressed in wing 

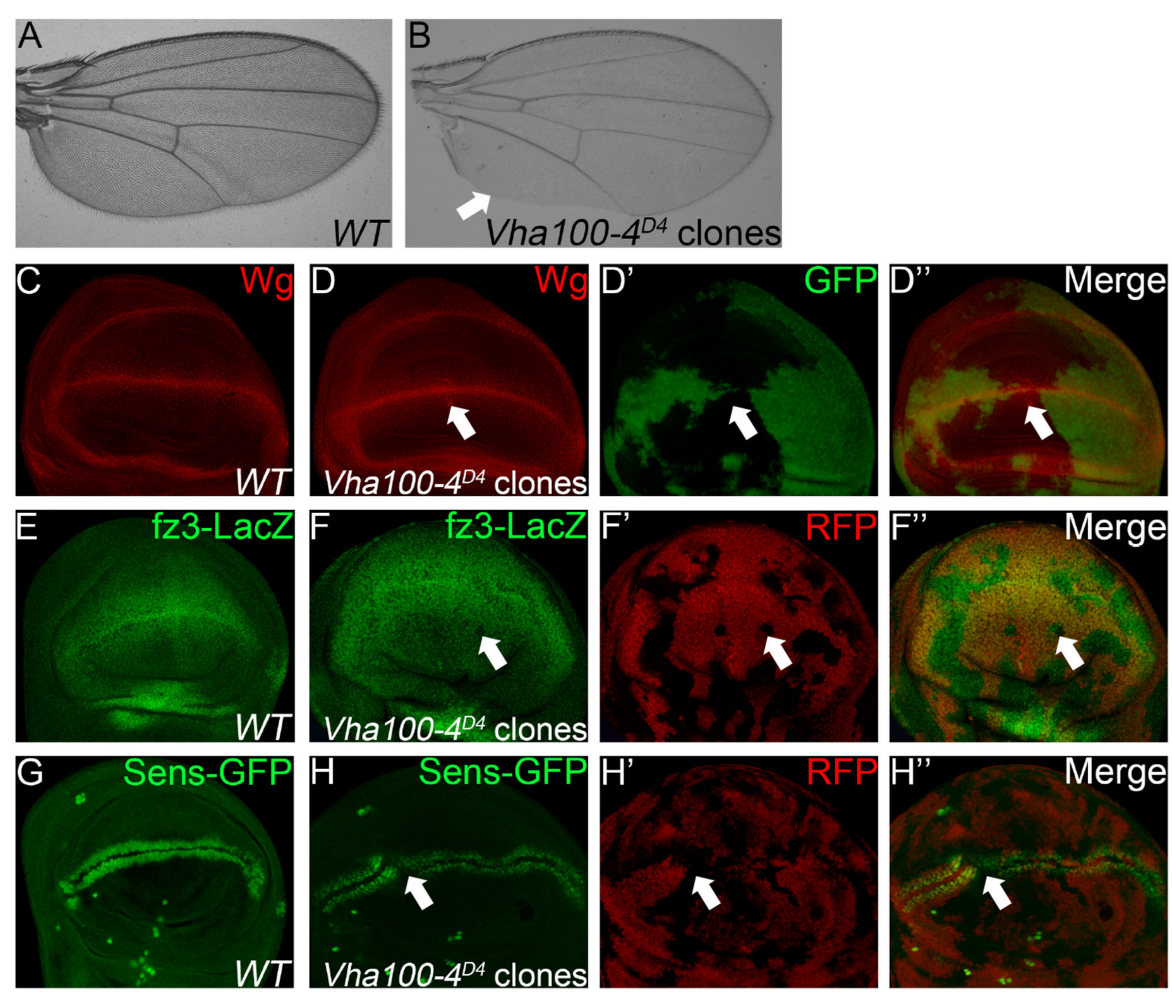

FIGURE 4 | Vha100-4 is required for proper activation of the Wg signaling pathway. (A,B) The adult wing of $W^{1118}$ stock is used as wild type control (A). Wing margin notches and marginal bristle loss are observed in homozygous Vha100-4 ${ }^{\text {4 }}$ mutant clones (B). (C,D) Immunostaining shows that Wg (red) is expressed by cells at the DN boundary in the pouch of wild type wing disk (C). Wg proteins (red) are accumulated in a subset of mutant $V h a 100-4^{D 4}$ clones $(n=7 / 13$; $\mathbf{D})$. (E,F) The Wg signaling reporter $f z 3-L a c Z$ (green) is expressed by all cells in the pouch area of wing disk (E). Expression of $f z 3-L a c Z$ (green) is dampened in $V h a 100-4 D 4$ mutant clones $(n=6 / 11 ; \mathbf{F})$. (G,H) Sens-GFP (green) is expressed in two rows of cells which are above and below the DN boundary (G). Expression of the Sens-GFP reporter (green) was inhibited in a subset of Vha100-4 ${ }^{D 4}$ mutant clones $(n=7 / 12 ; \mathbf{H})$. The mutant clones are marked by absence of GFP (D) or RFP (F,H) Representative mutant clones are indicated by arrows. Scale bars: $0.5 \mathrm{~mm}$ in $\mathbf{( A , B )}$ and $10 \mu \mathrm{m}$ in $\mathbf{( C - H )}$.

disk cells. Compared with the neighboring wild type cells, the expression level of Vha100-2 were significantly down-regulated in Vha100-2 $2^{D 2}$ homozygous cells (Figure 2D). The expression of Vha100-4 were also obviously decreased in Vha100-4 ${ }^{D 4}$ clones (Figure 2E).

\section{Vha100-2 Is Specifically Required for Wing Cuticle Formation}

We first examined the overall requirement of V-ATPase activity during wing development, and the contributions of distinct Vha100 isoforms were further dissected. Two mutants of the regulatory C subunit Vha44 were tested (Supplementary Figure S3A) and both of them resulted in various developmental defects including loss of wing marginal bristles and nicking of wing margin (Supplementary Figures S3B-D). We then investigated whether Vha44 regulates Notch (N) and Wingless (Wg) signaling activity, two major signaling pathways that were affected by mutations of other V-ATPase subunits (Vaccari et al., 2010; Kobia et al., 2013; Gleixner et al., 2014; Portela et al., 2018; Ren et al., 2018). In the wild type imaginal wing disk, the $\mathrm{N}$ target gene cut is produced in a narrow stripe of cells along the dorsal/ventral (D/V) boundary (Supplementary Figure S3E). In Vha44 ${ }^{K 05440}$ mutant clones, the expression of Cut is abolished when the clones are located at the D/V boundary (Supplementary Figure S3F). Clones of Vha44 mutant cells also displayed various degrees of accumulation of the ligand molecule Delta (Dl) and N protein itself, which were detected as intracellular puncta (Supplementary Figures S3G-J). During fly wing development, the Wg protein is generated at the D/V boundary (Supplementary Figure S3K) and transported throughout the wing disk to activate down-stream targets (Swarup and Verheyen, 2012). In Vha44 mutant cells, the expression level of $\mathrm{Wg}$ was not significantly affected, while the distribution of $\mathrm{Wg}$ protein in signal receiving cells was altered (Supplementary Figure S3L). As a consequence, the activity of $\mathrm{Wg}$ signaling was dampened in Vha44 mutant cells (Supplementary Figures S3M,N), which was monitored by a $f z 3$-LacZ reporter (Sato et al., 1999). In addition, we found that Vha44 ${ }^{K 05440}$ mutant cells displayed aberrant cellular cortex morphology (Supplementary Figures S3O,P) and likely underwent apoptosis (Supplementary Figures S3Q,R).

In contrast to the Vha44 mutants, fly wings harboring homozygous Vha100-2 $2^{D 2}$ mutant clones displayed wrinkles in the wing surface, without disruption of the vein patterns 
and wing margin integrity (Figures 3A,B). In agreement with the lack of patterning defects in the adult wing, the expression of Cut remained unaltered in Vha100-2 $2^{2}$ mutant clones (Supplementary Figure S4A). The expression level and distribution of $\mathrm{Wg}, \mathrm{N}$, and $\mathrm{Dl}$ proteins were also normal in Vha100-2 $2^{D 2}$ mutant cells (Supplementary Figures S4BD), suggesting that Vha100-2 is not required for these two pathways. Furthermore, phalloidin staining showed that Vha100-2 $2^{D 2}$ mutant had little influence for cell morphology (Supplementary Figure S4E). Wrinkling of the wing surface is often associated with defective cuticle deposition (Moussian, 2010). To further characterize the role of Vha100-2 on wing cuticle formation, we analyzed the cuticle impermeability by FB28 and Eosin Y staining (Wang et al., 2016, 2017). Wildtype wings with an intact cuticle layer is almost resistant to Eosin Y penetration (Figure 3A). By contrast, wings harboring homozygous Vha100-2 $2^{D 2}$ mutant clones showed patches of Eosin Y staining (Figure 3B). Similarly, a polysaccharide-specific dye FB28 was able to penetrate through the cuticle of wings bearing Vha100-2 ${ }^{D 2}$ mutant clones (Figures 3C,D). We further confirmed the role of Vha100-2 in cuticle integrity by knockdown of Vha100-2 expression in the posterior compartment of the wing. Regional specific inhibition of Vha100-2 led to wrinkles in the RNAi expressing area as well as diffusion of the FB28 dye (Figure 3E). Consistent with the adult wing phenotype, RNAi knock-down led to attenuation of Vha100-2 mRNA level when examined in the wing disk (Figure 3F). Taken together, our results suggest that Vha100-2 might be involved in cuticle formation of the wing.

\section{Vha100-4 Is Required for Proper Activation of the Wg Signaling Pathway}

When Vha100-4 ${ }^{D 4}$ mutant clones were induced in the wing, marginal notches and bristle loss were observed (Figures 4A,B). These marginal defects are typically resulted from disruption of $\mathrm{N}$ or Wg signaling activity (Logan and Nusse, 2004). Interestingly, we found that neither the target Cut nor the signaling molecules ( $\mathrm{N}$ and $\mathrm{Dl}$ ) were changed in Vha100-4 ${ }^{D 4}$ mutant cells (Supplementary Figure S5). On the other hand, aggregation of $\mathrm{Wg}$ proteins were observed in some mutant cells (Figures 4C,D), and the expression of the $\mathrm{Wg}$ activity reporter $f z 3$-LacZ (Figures 4E,F), and sens-GFP (Figures 4G,H) were dampened in a subset of Vha100-4 ${ }^{D 4}$ mutant clones. Collectively, these findings provide evidence that Vha100-4 is likely involved in Wg signaling activation during fly wing development.

\section{Vha100-1 Performs Redundant Function With Vha100-4}

Although previous studies suggest that Vha100-1 functions in the neuronal system to regulate fly development (Hiesinger et al., 2005; Wang et al., 2014), we noticed that ectopic veins were formed in wings bearing Vha100-1 $1^{1}$ mutant clones (Figures 5A,B). However, $\mathrm{N}$ and $\mathrm{Wg}$ signaling were not affected in Vha100-1 ${ }^{1}$ mutant cells (Supplementary Figures S6A,B). In addition, we noticed that Vha100-1 $1^{1}$ mutant cells did not undergo apoptosis (Supplementary Figure S6C) and displayed
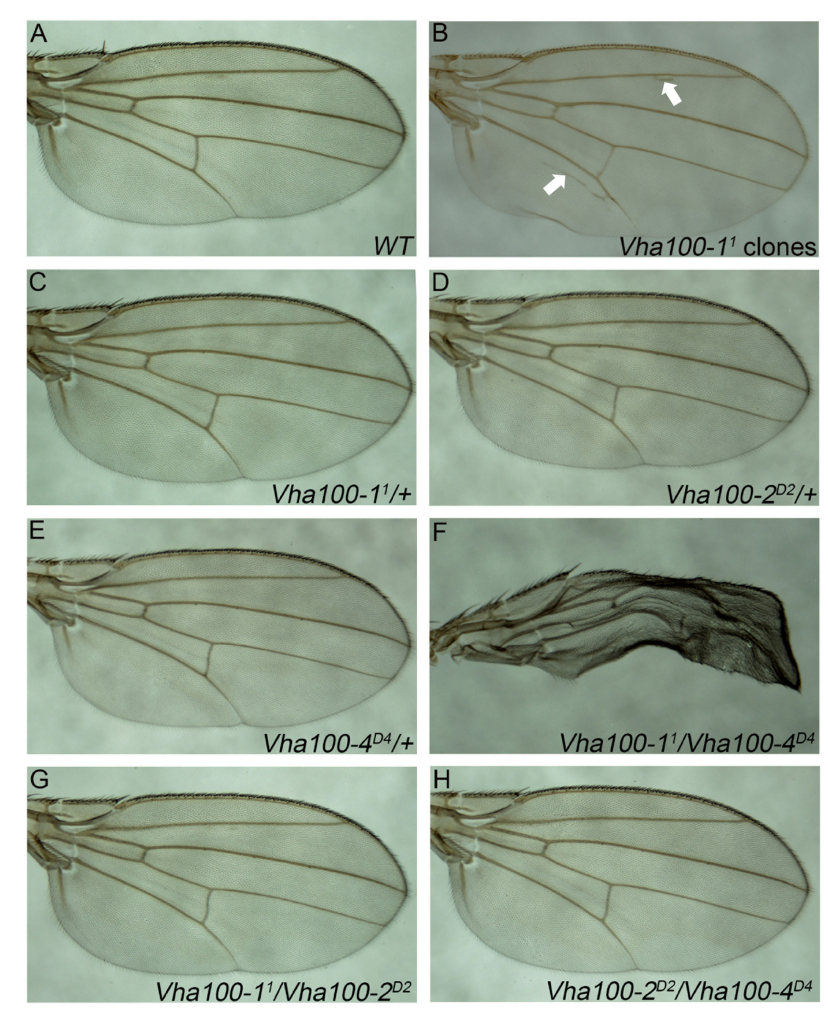

FIGURE 5 | Vha100-1 and Vha100-4 perform redundant functions during fly wing development. (A,B) The adult wing of $W^{1118}$ stock is used as wild type control (A). Ectopic veins (marked by arrows) are formed in wings bearing Vha100-11 mutant clones (B). (C-H) Compensation tests among Vha100-1, Vha100-2, and Vha100-4. The adult wings of heterozygous Vha100-1 ${ }^{1}$ (C), Vha100-2 ${ }^{D 2}$ (D), and Vha100-4 ${ }^{D 4}$ (E) flies are indistinguishable from wild type controls. Trans-heterozygous Vha100-1 ${ }^{1}$ Nha100-4 ${ }^{D 4}$ flies show severe wing developmental defects (F). Wings of Vha100-2D2/Vha100-11 (G) and Vha100-2 $2 /$ Vha100-4 ${ }^{\mathrm{D}}$ (H) flies display normal developmental patterns. Scale bars: $0.5 \mathrm{~mm}$.

normal cell morphology (Supplementary Figure S6D). The overall patterning and wing margin integrity was not affected by Vha100-1 ${ }^{1}$ mutant clones, suggesting that Vha100-1 might function in specific processes during vein cell differentiation.

Mutations of Vha100-1, Vha100-2, and Vha100-4 were unable to fully recapture the wing defects caused by Vha44 mutant, despite that they are all essential genes required for normal development. These results indicate that these isoforms may function redundantly and the loss of single isoform can be compensated by the remaining family members in certain developmental contexts. To test this hypothesis, we crossed Vha100-1 ${ }^{1}$, Vha100-2 ${ }^{D 2}$, and Vha100-4 ${ }^{D 4}$ flies with each other and examined the wing phenotypes in the trans-heterozygous progenies. For each mutant allele, the heterozygotes were indistinguishable with wild type controls (Figures 5C-E). The wings of Vha100-1 $1 /$ Vha100- $4^{D 4}$ flies were severely malformed (Figure 5F), indicating a strong genetic interaction and functional redundancy between Vha100-1 and Vha100-4. Combination of Vha100-2 $2^{2}$ with either Vha100-1 $1^{1}$ (Figure 5G) or Vha100-4 $4^{D 4}$ (Figure 5H) 
shows no effect on fly development and wing morphology, suggesting that Vha100-2 likely functions independently of the other two isoforms.

\section{DISCUSSION}

The V-ATPases are highly conserved multi-subunit pumps that transport hydrogen ions in exchange for ATP. Present in the endo-membranes of all eukaryotic cells, they are well known regulators for acidification of various intracellular compartments (Forgac, 2007). Studies in the larval midgut of the tobacco hornworm Manduca sexta are among the first reports to reveal that V-ATPases also pump protons across the plasma membranes of many specialized animal cells (Wieczorek et al., 2009). The great diversity of functions that the V-ATPases serve in eukaryotic organisms is recognized as a remarkable feature (Forgac, 2007; Wieczorek et al., 2009). Several subunits of the V-ATPases are encoded by multiple isoforms, and tissue specific expression of different isoforms have been demonstrated as a common strategy to fulfill the diverse requirement for V-ATPases (Toei et al., 2010). However, whether different isoforms are used in the same tissue to execute distinct functions is still an open question.

Here we report that isoforms of the V-ATPase a subunit are differentially required for Drosophila wing development. We provide genetic evidence that three of the five Vha100 isoforms (Vha100-1, Vha100-2, and Vha100-4) are involved in fly wing development. Somatic clonal analysis show that Vha100-1 regulates vein formation, while Vha100-2 functions in cuticle deposition and Vha100-4 participates in Wg signaling transduction. It is likely that Vha100-1 regulates vein formation through pathways transduced by other signaling molecules, such as bone morphogenetic protein (BMP), and epidermal growth factor (EGF) during fly wing development (Blair, 2007). Our results strongly support a model that V-ATPases might exist as multiple subtypes composed by diverse subunit isoforms in the same cells to meet the diversified demands. The accumulation of signaling molecules and alteration of signaling activities are not always fully penetrant in Vha100 mutant cells, which is also noticed in previous studies on other V-ATPase subunits (Yan et al., 2009; Vaccari et al., 2010; Ren et al., 2018). During tissue development, the V-ATPase regulates acidification of cellular organelles which are necessary for protein sorting, trafficking, and turnover (Forgac, 2007). It is conceivable that disrupting V-ATPase activity results in aberrant trafficking and degradation of signaling molecules, a highly dynamic process that might be only partially captured by conventional genetic analysis. In addition, our compensation tests suggest that beyond their specialized roles, Vha100-1, and Vha100-4 likely function redundantly for wing patterning and growth. This redundancy may also help to explain the minor defects observed when Vha100-1 and Vha100-4 single mutant clones were induced in the wing. Our observations suggest that the V-ATPases subtypes are able to constitute a coordinated network in cells. Further studies are required to dissect the exact mechanisms underlying the mode of action for V-ATPases.
Previous studies using RNAi knock-down experiments indicate that Vha100-2 is involved in the development of multiple fly tissues (Wang et al., 2014; Overend et al., 2016; Mondragon et al., 2019; Nagata et al., 2019). We find that Vha100-2 is the sole isoform required for wing cuticle integrity. This is the first report regarding a role of V-ATPases in insect cuticle formation. The cuticle is a multifunctional tissue that covers the whole body of insects, which provides significant protections from life threatening dangers such as dehydration, predators and pathogen infection (Moussian, 2010). It is possible that $\mathrm{V}$-ATPases might regulate membrane trafficking and secretion of the cuticle components in wing epithelia cells in a similar fashion as reported in Caenorhabditis elegans (Liégeois et al., 2007). However, V-ATPases may as well function in other steps during cuticle formation, which needs to be further explored.

\section{DATA AVAILABILITY STATEMENT}

The raw data supporting the conclusions of this article will be made available by the authors, without undue reservation, to any qualified researcher.

\section{AUTHOR CONTRIBUTIONS}

DM performed the mosaic screen and identified the Vha44 mutants. DM, YC, and NJ performed the immunostaining analyses. JZ performed the mRNA in situ hybridization. DM, YC, NJ, and JZ performed the statistical analyses for wing phenotypes. DM prepared the original draft. JS and JZ reviewed and edited the manuscript. JS and JZ supervised the project. All authors approved the final manuscript submitted for publication.

\section{FUNDING}

This work was supported by the National Natural Science Foundation of China (31772526 and 31970478 to JZ and 31872295 to JS).

\section{ACKNOWLEDGMENTS}

We thank Drs. Alan Jian Zhu, Yun Zhao, Renjie Jiao, Tao Wang, Wei $\mathrm{Wu}$, the Bloomington Stock Center, the Kyoto Fly Stock Center, the TsingHua Fly Stock Center, and the Developmental Studies Hybridoma Bank (DSHB) for fly stocks and antibodies. We thank Drs. Yinqiao Zhang and Zhenyong Du for help with construction of the phylogenetic tree.

\section{SUPPLEMENTARY MATERIAL}

The Supplementary Material for this article can be found online at: https://www.frontiersin.org/articles/10.3389/fgene.2020. 00723/full\#supplementary-material 


\section{REFERENCES}

Allan, A. K., Juan, D., Davies, S. A., and Julian, A. T. D. (2005). Genome-wide survey of V-ATPase genes in Drosophila reveals a conserved renal phenotype for lethal alleles. Physiol. Genom. 22, 128-138. doi: 10.1152/physiolgenomics. 00233.2004

Bassett, A. R., Tibbit, C., Ponting, C. P., and Liu, J. L. (2013). Highly efficient targeted mutagenesis of Drosophila with the CRISPR/Cas9 system. Cell Rep. 4, 220-228. doi: 10.1016/j.celrep.2013.06.020

Blair, S. S. (2007). Wing vein patterning in Drosophila and the analysis of intercellular signaling. Annu. Rev. Cell Dev. Biol. 23, 293-319. doi: 10.1146/ annurev.cellbio.23.090506.123606

Buechling, T., Bartscherer, K., Ohkawara, B., Chaudhary, V., Spirohn, K., Niehrs, C., et al. (2010). Wnt/Frizzled signaling requires dPRR, the Drosophila homolog of the prorenin receptor. Curr. Biol. 20, 1263-1268. doi: 10.1016/j.cub.2010.05. 028

Cipriano, D. J., Wang, Y., Bond, S., Hinton, A., Jefferies, K. C., Qi, J., et al. (2008). Structure and regulation of the vacuolar ATPases. Biochim. Biophys. Acta 1777, 599-604.

Forgac, M. (2007). Vacuolar ATPases: rotary proton pumps in physiology and pathophysiology. Nat. Rev. Mol. Cell Biol. 8, 917-929. doi: 10.1038/nrm2272

Gleixner, E. M., Canaud, G., Hermle, T., Guida, M. C., Kretz, O., Huber, T. B., et al. (2014). V-ATPase/ mTOR signaling regulates megalin-mediated apical endocytosis. Cell Rep. 8, 10-19. doi: 10.1016/j.celrep.2014.05.035

Gratz, S. J., Ukken, F. P., Rubinstein, C. D., Thiede, G., Donohue, L. K., Cummings, A. M., et al. (2014). Highly specific and efficient CRISPR/Cas9-catalyzed homology-directed repair in Drosophila. Genetics 196, 961-971. doi: 10.1534/ genetics.113.160713

Hermle, T., Saltukoglu, D., Grucnewald, J., Walz, G., Simons, M., Gru, J., et al. (2010). Regulation of Frizzled-dependent planar polarity signaling by a V-ATPase subunit. Curr. Biol. 20, 1269-1276. doi: 10.1016/j.cub.2010.05.057

Hiesinger, P. R., Fayyazuddin, A., Mehta, S. Q., Rosenmund, T., Schulze, K. L., Zhai, R. G., et al. (2005). The v-ATPase V0 subunit al is required for a late step in synaptic vesicle exocytosis in Drosophila. Cell 121, 607-620. doi: 10.1016/j.cell. 2005.03.012

Hinton, A., Bond, S., and Forgac, M. (2009). V-ATPase functions in normal and disease processes. Pflugers Arch. 457, 589-598. doi: 10.1007/s00424-00 7-0382-4

Inoue, T., and Forgac, M. (2005). Cysteine-mediated cross-linking indicates that subunit $\mathrm{C}$ of the V-ATPase is in close proximity to subunits $\mathrm{E}$ and $\mathrm{G}$ of the V1 domain and subunit a of the V0 domain. J. Biol. Chem. 280, 27896-27903. doi: 10.1074/jbc.m504890200

Inoue, T., Wang, Y., Jefferies, K., Qi, J., Hinton, A., and Forgac, M. (2005). Structure and regulation of the V-ATPases. J. Bioenerg. Biomembr. 37, 393-398.

Jianhua, Z., Samir, B., and John, L. R. (2015). Electron cryomicroscopy observation of rotational states in a eukaryotic V-ATPase. Nature 521, 241-245. doi: 10. $1038 /$ nature 14365

Julian, A. T. D. (1999). The multifunctional Drosophila melanogaster V-ATPase is encoded by a multigene family. J. Bioenerg. Biomembr. 31, 75-83.

Kobia, F., Duchi, S., Deflorian, G., and Vaccari, T. (2013). Pharmacologic inhibition of vacuolar HC ATPase reduces physiologic and oncogenic Notch signaling. Mol. Oncol. 8, 207-220. doi: 10.1016/j.molonc.2013.11.002

Krogh, A., Larsson, B., von Heijne, G., and Sonnhammer, E. L. (2001). Predicting transmembrane protein topology with a hidden Markov model: application to complete genomes. J. Mol. Biol. 305, 567-580. doi: 10.1006/jmbi.2000. 4315

Leng, X. H., Manolson, M., and Forgac, M. (1998). Function of the COOH-terminal domain of Vph1p in activity and assembly of the yeast V-ATPase. J. Biol. Chem. 273, 6717-6723. doi: 10.1074/jbc.273.12.6717

Leng, X. H., Manolson, M., Liu, Q., and Forgac, M. (1996). Site-directed mutagenesis of the $100-\mathrm{kDa}$ subunit (Vphlp) of the yeast vacuolar $(\mathrm{H}+\square)$ ATPase. J. Biol. Chem. 271, 22487-22493. doi: 10.1074/jbc.271.37.22487

Liégeois, S., Benedetto, A., Michaux, G., Belliard, G., and Labouesse, M. (2007). Genes required for osmoregulation and apical secretion in Caenorhabditis elegans. Genetics 175, 709-724. doi: 10.1534/genetics.106.066035

Logan, C. Y., and Nusse, R. (2004). The Wnt signaling pathway in development and disease. Annu. Rev. Cell Dev. Biol. 20, 781-810.
Minh, B. Q., Nguyen, M. A. T., and von Haeseler, A. (2013). Ultrafast approximation for phylogenetic bootstrap. Mol. Biol. Evol. 30, 1188-1195. doi: 10.1093/molbev/mst024

Mondragon, A. A., Yalonetskaya, A., Ortega, A. J., Elguero, J., Chung, W. S., and McCall, K. (2019). Lysosomal machinery drives extracellular acidification to direct non- apoptotic cell death. Cell Rep. 27, 11-19.

Moussian, B. (2010). Recent advances in understanding mechanisms of insect cuticle differentiation. Insect. Biochem. Mol. Biol. 40, 363-375. doi: 10.1016/ j.ibmb.2010.03.003

Nagata, R., Nakamura, M., Sanaki, Y., and Igaki, T. (2019). Cell competition is driven by autophagy. Dev. Cell. 51, 99-112.

Nelson, N. (2003). A journey from mammals to yeast with vacuolar H+-ATPase (V-ATPase). J. Bioenerg. Biomembr. 35, 281-289.

Nishi, T., and Forgac, M. (2000). Molecular cloning and expression of three isoforms of the $100-\mathrm{kDa}$ a subunit of the mouse vacuolar proton-translocating ATPase. J. Biol. Chem. 275, 6824-6830. doi: 10.1074/jbc.275.10.6824

Overend, G., Luo, Y., Henderson, L., Douglas, A. E., Davies, S. A., and Dow, J. A. (2016). Molecular mechanism and functional significance of acid generation in the Drosophila midgut. Sci. Rep. 6:27242.

Petzoldt, A. G., Gleixner, E. M., Fumagalli, A., Vaccari, T., and Simons, M. (2013). Elevated expression of the V-ATPase C subunit triggers JNK-dependent cell invasion and overgrowth in a Drosophila epithelium. Dis. Model. Mech. 6, 689-700. doi: 10.1242/dmm.010660

Portela, M., Yang, L., Paul, S., Li, X., Veraksa, A., Parsons, L. M., et al. (2018). Lgl reduces endosomal vesicle acidification and Notch signaling by promoting the interaction between Vap33 and the V-ATPase complex. Sci. Signal. 11:eaar1976. doi: 10.1126/scisignal.aar1976

Qi, J., Wang, Y., and Forgac, M. (2007). The vacuolar (H+)-ATPase: subunit arrangement and in vivo regulation. J. Bioenerg. Biomembr. 39, 423-426. doi: 10.1007/s10863-007-9116-8

Ren, L., Mo, D., Li, Y., Liu, T., Yin, H., Jiang, N., et al. (2018). A genetic mosaic screen identifies genes modulating Notch signaling in Drosophila. PLoS One 13:e0203781. doi: 10.1371/journal.pone.0203781

Sarov, M., Barz, C., Jambor, H., Hein, M. Y., Schmied, C., Suchold, D., et al. (2016). A genome-wide resource for the analysis of protein localisation in Drosophila. eLife 5:e12068.

Sato, A., Kojima, T., Ui-Tei, K., Miyata, Y., and Saigo, K. (1999). Dfrizzled-3, a new Drosophila Wnt receptor, acting as an attenuator of Wingless signaling in wingless hypomorphic mutants. Development 126, 4421-4430.

Saw, N. M., Kang, S. Y., Parsaud, L., Han, G. A., Jiang, T., Grzegorczyk, K., et al. (2011). Vacuolar H(+)-ATPase subunits Voa1 and Voa2 cooperatively regulate secretory vesicle acidification, transmitter uptake, and storage. Mol. Biol. Cell. 22, 3394-3409. doi: 10.1091/mbc.e11-02-0155

Su, Y., Ospina, J. K., Zhang, J., Michelson, A. P., Schoen, A. M., and Zhu, A. J. (2011). Sequential phosphorylation of smoothened transduces graded hedgehog signaling. Sci. Signal. 4:ra43. doi: 10.1126/scisignal.2001747

Swarup, S., and Verheyen, E. M. (2012). Wnt/Wingless signaling in Drosophila. Cold Spring Harb. Perspect. Biol. 4:a007930.

Theodosiou, N. A., and Xu, T. (1998). Use of FLP/FRT system to study Drosophila development. Methods 14, 355-365. doi: 10.1006/meth.1998.0591

Toei, M., Saum, R., and Forgac, M. (2010). Regulation and isoform function of the V-ATPases. Biochemistry 49, 4715-4723. doi: 10.1021/bi100397s

Toyomura, T., Murata, Y., Yamamoto, A., Oka, T., Sun-Wada, G. H., Wada, Y., et al. (2003). From lysosomes to the plasma membrane: localization of vacuolar-type $\mathrm{H}+$-ATPase with the a3 isoform during osteoclast differentiation. J. Biol. Chem. 278, 22023-22030. doi: 10.1074/jbc.m3024 36200

Toyomura, T., Oka, T., Yamaguchi, C., Wada, Y., and Futai, M. (2000). Three subunit a isoforms of mouse vacuolar $\mathrm{H}(+)$-ATPase. Preferential expression of the $a 3$ isoform during osteoclast differentiation. J. Biol. Chem. 275, 8760-8765. doi: $10.1074 /$ jbc. 275.12 .8760

Trifinopoulos, J., Nguyen, L.-T., von Haeseler, A., and Minh, B. Q. (2016). W-IQTREE: a fast online phylogenetic tool for maximum likelihood analysis. Nucleic Acids Res. 44, W232-W235.

Vaccari, T., Duchi, S., Cortese, K., Tacchetti, C., and Bilder, D. (2010). The vacuolar ATPase is required for physiological as well as pathological activation of the Notch receptor. Development 137, 1825-1832. doi: 10.1242/dev.045484 
Wang, D., Epstein, D., Khalaf, O., Srinivasan, S., Williamson, W. R., Fayyazuddin, A., et al. (2014). Ca2+-Calmodulin regulates SNARE assembly and spontaneous neurotransmitter release via v-ATPase subunit V0 a1. J. Cell Biol. 205, 21-31. doi: $10.1083 /$ jcb.201312109

Wang, J., Kean, L., Yang, J., Allan, A. K., Davies, S. A., Herzyk, P., et al. (2004). Function-informed transcriptome analysis of Drosophila renal tubule. Genome Biol. 5:R69.

Wang, Y., Carballo, R. G., and Moussian, B. (2017). Double cuticle barrier in two global pests, the whitefly Trialeurodes vaporariorum and the bedbug Cimex lectularius. J. Exp. Biol. 220, 1396-1399. doi: 10.1242/jeb.156679

Wang, Y., Yu, Z., Zhang, J., and Moussian, B. (2016). Regionalization of surface lipids in insects. Proc. Biol. Sci. 283:20152994. doi: 10.1098/rspb.2015.2994

Wieczorek, H., Beyenbach, K. W., Huss, M., and Vitavska, O. (2009). Vacuolar-type proton pumps in insect epithelia. J. Exp. Biol. 212, 1611-1619. doi: 10.1242/jeb. 030007

Wilkens, S., Inoue, T., and Forgac, M. (2004). Three-dimensional structure of the vacuolar ATPase: localization of subunit $\mathrm{H}$ by difference imaging and chemical cross-linking. J. Biol. Chem. 279, 41942-41949. doi: 10.1074/jbc.m4078 21200

Williamson, W. R., Wang, D., Haberman, A. S., and Hiesinger, P. R. (2010a). A dual function of V0-ATPase al provides an endolysosomal degradation mechanism in Drosophila melanogaster photoreceptors. J. Cell Biol. 189, 885-899. doi: $10.1083 /$ jcb. 201003062
Williamson, W. R., Yang, T., Terman, J. R., and Hiesinger, P. R. (2010b). Guidance receptor degradation is required for neuronal connectivity in the Drosophila nervous system. PLoS Biol. 8:e1000553. doi: 10.1371/journal.pone.01000553

Wissel, S., Harzer, H., Bonnay, F., Burkard, T. R., Neumüller, R. A., and Knoblich, J. A. (2018). Time-resolved transcriptomics in neural stem cells identifies a v-ATPase/Notch regulatory loop. J. Cell Biol. 217, 3285-3300. doi: 10.1083/jcb. 201711167

Xu, T., and Rubin, G. M. (1993). Analysis of genetic mosaics in developing and adult Drosophila tissues. Development 117, 1223-1237.

Yan, Y., Denef, N., and Schucpbach, T. (2009). The vacuolar proton pump, VATPase, is required for notch signaling and endosomal trafficking in Drosophila. Dev. Cell. 17, 387-402. doi: 10.1016/j.devcel.2009.07.001

Conflict of Interest: The authors declare that the research was conducted in the absence of any commercial or financial relationships that could be construed as a potential conflict of interest.

Copyright (C) 2020 Mo, Chen, Jiang, Shen and Zhang. This is an open-access article distributed under the terms of the Creative Commons Attribution License (CC BY). The use, distribution or reproduction in other forums is permitted, provided the original author(s) and the copyright owner(s) are credited and that the original publication in this journal is cited, in accordance with accepted academic practice. No use, distribution or reproduction is permitted which does not comply with these terms. 